\title{
Electron Auto-Localization Tailored by Its Thermal Energy: Dynamic Matrix Approach (DMA)
}

\author{
Idriss Fomadjo Fokou ${ }^{1 *}$, Michael Nana Jipdi1,2, Martin Tchoffo ${ }^{1,3}$, Lukong Cornelius Fai1 \\ ${ }^{1}$ Research Unit Condensed Matter, Electronics and Signal Processing, Université de Dschang, Dschang, Cameroon \\ ${ }^{2}$ Department of Physics, Higher Teacher Training College, University of Bamenda, Bambili, Cameroon \\ ${ }^{3}$ Centre d'Études et de Recherches en Agronomie et en Biodiversité, Faculté d'Agronomie et des Sciences Agricoles, \\ University of Dschang, Dschang, Cameroon \\ Email: jmichaelnicky@yahoo.fr, ^fokouidriss@yahoo.fr
}

How to cite this paper: Fokou, I.F., Jipdi, M.N., Tchoffo, M. and Fai, L.C. (2021) Electron Auto-Localization Tailored by Its Thermal Energy: Dynamic Matrix Approach (DMA). Journal of Applied Mathematics and Physics, 9, 515-527.

https://doi.org/10.4236/jamp.2021.93036

Received: February 6, 2021

Accepted: March 28, 2021

Published: March 31, 2021

Copyright $\odot 2021$ by author(s) and Scientific Research Publishing Inc. This work is licensed under the Creative Commons Attribution International License (CC BY 4.0).

http://creativecommons.org/licenses/by/4.0/

\begin{abstract}
This paper investigates the thermal energy effect on electron auto-localization. The polaron characteristics (self-action potential and effective mass) are observed to be expressed via the renormalized electron-phonon coupling constant tailored by the thermal energy. Low temperatures are observed to favour auto-localization of the carrier while high temperatures favour polaron undressing and subsequent quenching of the quantum behaviour thereby rendering the system classical. The critical (transition) temperature $\tau_{c}$ expressed via the critical coupling constant $\Upsilon_{C}$ is found to be the separating boundary between the quantum and the classical phases. Therefore, the polaron undergoes phase transition (from self-tapped to quasi free states) when the temperature of the medium is enhanced.
\end{abstract}

\section{Keywords}

Autolocalization, Quantum Phase Transition, Self-Trapping, Critical Temperature

\section{Introduction}

Self-trapping relates local perturbation-like defects, impurity as well as regions of high affinity in amorphous solids and has been observed for electrons, holes, excitons, muons as well as muonium [1] [2] [3]. The phenomenon has also been observed for conduction electrons producing a local distortion and polarization of the lattice. Various experimental results demonstrate anomalous manifesta- 
tion of the self-trapping effect in bulk crystals [4] however, theoretical investigations are still going on in understanding such strange manifest. It is well known that a conduction electron (or hole) together with its self-induced polarization in a polar semiconductor or an ionic crystal results in a quasi-particle called a polaron. The physical property of the polaron differs from those of the band-electron and in particular is characterized by its binding or self-energy, effective mass and by its response to external electric and magnetic fields. The general polaron concept was proposed by L.D. Landau [5] [6] and subsequently Landau and Solomon Pekar [7] [8] [9] as the auto-localization of an electron in an ideal crystal as a result of a lattice deformation by the field induced by the electron where the local states are assumed to be immobile. L.D. Landau [5] [6] investigated the polaron characteristics (self-energy and effective mass) in what Fröhlich [10] demonstrated to correspond an adiabatic or strong-coupling regime.

The polaron concept is of interest, not only for its description of the particular physical properties of charge carriers in polarizable solids but also due to its interesting field theoretical model consisting of a fermion interacting with a scalar bosonic field. Many studies have been devoted to electron auto-localization that is achieved when the polaron ground-state energy equals that of the quasi-free state [11] [12] [13] [14] [15] with the signature being the critical coupling constant above which the electron is self-trapped. Recent investigations show that polaron characteristics may decrease with temperature [16] [17] [18] [19] and a transition from a quantum phase to classical phase predicted [19]. One of the prominent results is seen in ref [20] where the authors investigate experimentally the mechanisms of energy transfer in $\mathrm{Mn}^{2+}$ doped ethylammonium lead bromide. It is observed that for large temperature, photoluminescence is dominated by emission from $\mathrm{Mn}^{2+}$, with a complete suppression of band edge emission and self-trapped exciton emission. However, for low temperature in addition to $\mathrm{Mn}^{2+}$ emission, photoluminescence is observed from band edge and self-trapped exciton emission; this potentially confirms the existence of self-trapping at low temperature.

Nevertheless, many authors strongly believe that, for a large class of (generalized Fröhlich) models no such transition exists. More precisely, the dimensionality of space has no qualitative influence on phase transition [21]. From another perspective, it is seen that, the phase transition from the mobile (quasi-free) to the localized polaron state, found in the literature is a feature of approximation rather than an intrinsic property of the Fröhlich Hamiltonian [22]. However, the intrinsic modification in the parameter of the system can produce such a transition. Such disturbance can be produced by external field influence, thermal energy... In ref [23], the self-trapping energy of a magnetopolaron in a polar-crystal slab is investigated; it is observed that, the temperature dependence of the self-trapping energies is strongly dependent upon the strength of the magnetic field.

Therefore, polaron self-energy and effective mass in a polar crystal is strongly 
related to external field as well as thermal energy and so, the description of the polaron dynamics under an arbitrary temperature is of great interest. Several phenomena like high temperature superconductivity [24] are suspected to be related to bipolarons (the stable pair of coupled polarons). Many studies are devoted to the behaviours of these carriers under thermal energy with the conductivity and mobility being quantities of primary interest [25] [26]. It is observed that the quasi-particles exist both in self-trapped (quantum phase) and quasi-free state (classical phase); however, the limit separating quantum phase and classical phase is not determined.

This paper aims to demonstrate theoretically that polaron self-trapping transition can be induced by thermal energy and derive the critical temperature separating the quantum and the classical phase. The work is organized as follows: section II applies the Dynamic Matrix Approach (DMA) in the derivation of the polaron energy momentum relation for arbitrary thermal energy in the strong coupling regime; Section III focuses on the results and discussions of the polaron characteristics at low and high temperatures. Section IV, is the summary and conclusion.

\section{Energy-Momentum (E-P) Relation for an Arbitrary Temperature}

We consider the polaron due to the interaction of the electron (or hole) with the long-wavelength optical phonons described by the Fröhlich field-theoretical Hamiltonian:

$$
H=\frac{P^{2}}{2 m}+\sum_{Q} \hbar \omega_{L O} b_{Q}^{\dagger} b_{Q}+\sum_{Q} V_{Q} \mathrm{e}^{i Q r}\left(b_{Q}+b_{-Q}^{\dagger}\right)
$$

We apply the Dynamic matrix approach [27] [28], the so-called Jipdi-Fai-Tchoffo (JFT) approach which is an all coupling approach to investigate the polaron problem. The particle momentum and the coupling strength are tailored by the lattice distortions where the lattice polarization is allowed to relax or adapt to the electronic distribution:

$$
\boldsymbol{P} \rightarrow \boldsymbol{P}+\rho \boldsymbol{P}_{Q f} .
$$

Here, the fluctuation strength $\rho$ tailors the quantum and classical behaviours of the carrier respectively for $\rho \neq 0$ and $\rho=0 . P_{Q f}$ is the momentum fluctuation in the local frame while $P$, the total momentum satisfying the following Heisenberg equation of motion:

$$
i \hbar \dot{P}=[H, P], i \hbar \dot{b}_{Q}=\left[H, b_{Q}\right], i \hbar \dot{b}_{Q}^{\dagger}=\left[H, b_{Q}^{\dagger}\right]
$$

From here,

$$
\begin{aligned}
\boldsymbol{P}= & \boldsymbol{P}_{0}+\sum_{Q}(1-\rho) \hbar \boldsymbol{Q}\left(a_{Q} \Phi_{Q}(\boldsymbol{r}, \tau)+a_{Q}^{\dagger} \Phi_{Q}^{*}(\boldsymbol{r}, \tau)\right) \\
& -\sum_{Q}(1-\rho) \hbar \boldsymbol{Q} \Phi_{Q}(\boldsymbol{r}, \tau) \Phi_{Q}^{*}(\boldsymbol{r}, \tau)+\rho \boldsymbol{P}_{Q f}
\end{aligned}
$$




$$
\boldsymbol{Q} \boldsymbol{r} \approx(1-\rho) \boldsymbol{Q} \boldsymbol{r}_{0}+(1-\rho)^{2} \frac{\hbar Q^{2}}{2 m_{0}}\left(2 n_{Q}+1\right)+(1-\rho)\left(1-C_{P_{0}}\right) \frac{\hbar \mathbf{P Q}}{m}+\rho \boldsymbol{r}_{Q f},
$$

with the relevant phonon distribution function:

$$
n_{Q}=\frac{1}{\exp \left(\frac{\hbar \omega_{L O}}{K_{B} T}\right)-1} \equiv \frac{1}{\exp \left(\frac{T_{D}}{T}\right)-1},
$$

where $T_{D}$ is the Debye temperature of the lattice. Considering

$$
\begin{aligned}
& \left\langle r_{Q f}\right\rangle=\left\langle p_{Q f}\right\rangle=0 \\
& \left\langle r_{Q f}^{2}\right\rangle=\frac{\hbar \varepsilon^{1 / 2}}{m \gamma_{Q}},\left\langle p_{Q f}^{2}\right\rangle=\frac{m \hbar \gamma_{Q} \varepsilon^{1 / 2}}{4}
\end{aligned}
$$

For simplicity, we consider that $\Phi_{Q}(\boldsymbol{r}, \tau) \equiv \Phi_{Q}$ then follows the Hamiltonian:

$$
\begin{aligned}
H_{0}= & \frac{P_{0}^{2}}{2 m_{0}}\left(1-C\left(P_{0}\right)\right)^{2}+\sum_{Q}\left(\hbar \omega_{L O}+\frac{\hbar^{2} Q^{2}}{2 m_{0}}(1-\rho)^{2}\right) \Phi_{Q} \Phi_{Q}^{*}+\rho^{2} \frac{\varepsilon^{1 / 2} \hbar \gamma_{Q}}{8} \\
& +\sum_{Q}\left(\hbar \omega_{L O}+(1-\rho)^{2} \frac{\hbar^{2} Q^{2}}{m_{0}} \Phi_{Q} \Phi_{Q}^{*}\right) a_{Q}^{?} a_{Q}+\sum_{Q} \eta_{Q}(\tau) a_{Q}+\eta_{Q}(\tau) a_{Q} \\
& +\sum_{Q} \exp \left(-\rho^{2} \frac{\varepsilon^{1 / 2} \hbar Q^{2}}{2 m \gamma_{Q}}\right)\left(V_{Q} \mathrm{e}^{i(1-\rho) Q r+i \omega_{Q} \tau} \Phi_{Q}+V_{Q}^{*} \mathrm{e}^{-i(1-\rho) Q r-i \omega_{Q} \tau} \Phi_{Q}^{*}\right)
\end{aligned}
$$

with

$$
\begin{gathered}
\eta_{Q}(\tau)=\frac{\boldsymbol{P}_{0}\left(1-C\left(P_{0}\right)\right)}{m} \hbar \boldsymbol{Q} \Phi_{Q}+\hbar \omega_{L O} \Phi_{Q}+V_{Q} \mathrm{e}^{i(1-\rho) \boldsymbol{Q} r+i \omega_{0 Q} \tau} \exp \left(-\rho^{2} \frac{\varepsilon^{1 / 2} \hbar Q^{2}}{2 m \gamma_{Q}}\right) \\
\Phi_{Q}=\frac{-V_{Q}^{*} \exp \left(-i(1-\rho) \boldsymbol{Q} \boldsymbol{r}_{0}-i \omega_{0 Q} \tau-\frac{\rho^{2} \varepsilon^{1 / 2} Q^{2} \hbar}{2 m \gamma_{Q}}\right)}{\omega_{0 Q}} \\
\omega_{0 Q}=\omega_{L O}+(1-\rho)^{2} \frac{\hbar Q^{2}}{2 m_{0}}\left(2 n_{Q}+1\right)-(1-\rho)\left(1-C_{P_{0}}\right) \frac{\boldsymbol{P Q}}{m}
\end{gathered}
$$

Here, $\varepsilon$ is the dimension of the system and $\gamma_{Q}$ a variational parameter. Letting

$$
H_{e f f}=E_{Q}\left(P_{0}\right)+\sum_{Q} \eta_{Q}(\tau) a_{Q}+\eta_{Q}^{*}(\tau) a_{Q}^{?}
$$

where

$$
\begin{aligned}
E_{Q}\left(P_{0}\right)= & \frac{P_{0}^{2}}{2 m_{0}}\left(1-C\left(P_{0}\right)\right)^{2}+\sum_{Q}\left(\hbar \omega_{L O}+\frac{\hbar^{2} Q^{2}}{2 m_{0}}(1-\rho)^{2}\right) \Phi_{Q} \Phi_{Q}^{*} \\
& +\sum_{Q} \exp \left(-\rho^{2} \frac{\varepsilon^{1 / 2} \hbar Q^{2}}{2 m \gamma_{Q}}\right)\left(V_{Q} \mathrm{e}^{i(1-\rho) Q r+i \omega_{0 Q} \tau} \Phi_{Q}+V_{Q}^{*} \mathrm{e}^{-i(1-\rho) Q r-i \omega_{0 Q} \tau} \Phi_{Q}^{*}\right) \\
& +\sum_{Q}\left(\hbar \omega_{L O}+(1-\rho)^{2} \frac{\hbar^{2} Q^{2}}{m_{0}} \Phi_{Q} \Phi_{Q}^{*}\right) a_{Q}^{?} a_{Q}+\rho \frac{\varepsilon^{1 / 2} \hbar \gamma_{Q}}{8}
\end{aligned}
$$

then from refs [27] [28] [29] [30] and Equation (9), we obtain the following 
transition matrix elements:

$$
T_{n l}=\exp \left(\frac{-i t}{\hbar} E_{Q}^{n, l}\left(P_{0}\right)\right) \mathrm{e}^{-\frac{1}{2} \lambda_{Q}} \frac{l !}{n !} \lambda_{Q}^{\frac{l-n}{2}} L_{n}^{l}\left(\lambda_{Q}\right) .
$$

Averaging the transision matrix by an arbitrary phonon state $\left|n_{Q}\right\rangle$, then we obtain the polaron energy:

$$
\begin{aligned}
& \epsilon_{n_{Q}}= \frac{P_{0}^{2}}{2 m_{0}}\left(1-C\left(P_{0}\right)\right)^{2}-\frac{1}{\hbar} \sum_{Q} \frac{\left|V_{Q}\right|^{2} \exp \left(-\rho^{2} \frac{\varepsilon^{1 / 2} \hbar Q^{2}}{2 m \gamma_{Q}}\right)}{\omega_{L O}+(1-\rho)^{2} \frac{\hbar Q^{2}}{2 m_{0}} S-\left(1-C\left(P_{0}\right)\right) \frac{P_{0} q}{m}} \\
&+\frac{1}{\hbar^{2}} \sum_{Q} \frac{\left(1-C\left(P_{0}\right)\right) \frac{P_{0} q}{m}\left|V_{Q}\right|^{2} \exp \left(-\rho^{2} \frac{\varepsilon^{1 / 2} \hbar Q^{2}}{2 m \gamma_{Q}}\right)}{\left(\omega_{L O}+(1-\rho)^{2} \frac{\hbar Q^{2}}{2 m_{0}} S-\left(1-C\left(P_{0}\right)\right) \frac{P_{0} q}{m}\right)^{2}} \\
&+\rho^{2} \frac{\varepsilon^{1 / 2} \hbar \gamma_{Q}}{8}+\hbar \omega_{L O} n_{Q} \\
& S=2 n_{Q}+1=\frac{1}{\tanh \left(\frac{T_{D}}{2 T}\right)} .
\end{aligned}
$$

Equation (11) give the energy momentum (E-P) relation of a polaron for an arbitrary thermal energy. The self-action potential is obtained at the minimum of the E-P curve while the effective mass is the inverse of the second derivative of that relation which respect to the momentum and measured at the energy minimum. The self-action potential energy of the system is therefore

$$
U_{\Upsilon_{v}}=\rho^{2} \frac{\varepsilon^{1 / 2} \hbar \gamma_{Q}}{8}-\frac{\hbar \omega_{L O} \Upsilon_{v}}{(1-\rho) S^{1 / 2}} e^{-\frac{2 \rho^{2} \varepsilon^{1 / 2} \omega_{L O}}{(1-\rho)^{2} S \gamma_{Q}}}\left(1+\operatorname{erf}\left(\frac{2^{1 / 2} \rho \varepsilon^{1 / 4} \omega_{L O}^{1 / 2}}{(1-\rho) S^{1 / 2} \gamma_{Q}^{1 / 2}}\right)\right)
$$

The weak coupling result is obtained for $\rho=0$ while the strong coupling result, for $\rho \neq 0$. The thermal energy is found to renormalize the electronphonon coupling constant $\Upsilon_{v}$ as

$$
\Upsilon_{\text {eff }}^{2}=\frac{\Upsilon_{v}^{2}}{S}=\Upsilon_{v}^{2} \tanh \frac{T_{D}}{2 T}
$$

For limiting values of low and high temperatures then we have respectively

$$
\lim _{T \rightarrow 0} \Upsilon_{\text {eff }}^{2}=\lim _{T \rightarrow 0} \Upsilon_{v}^{2} \tanh \frac{T_{D}}{2 T}=\Upsilon_{v}^{2}
$$

and

$$
\lim _{T \rightarrow+\infty} \Upsilon_{\text {eff }}^{2}=\lim _{T \rightarrow+\infty} \Upsilon_{v}^{2} \tanh \frac{T_{D}}{2 T}=0
$$

The renormalized electron-phonon coupling constant is observed to be tailored by the thermal energy where low temperatures favour auto-localization of the carrier while high temperatures favour polaron undressing and subsequent 
quenching of the quantum behaviour rendering the system obey classical laws.

\section{Results and Discussion: Low and High Temperature Regime}

\subsection{Weak-Coupling Limits}

Here, we consider self-action potential energy in (12) for the fluctuation strength $\rho=0$ and extremise the resultant expression with respect to the variational parameter $\gamma_{Q}$ then this yields for arbitrary thermal energy, the polaron self-action potential energy

$$
U_{\Upsilon_{v}}=-\hbar \omega_{L O} \Upsilon_{v} \tanh ^{\frac{1}{2}} \frac{T_{D}}{2 T}=-\hbar \omega_{L O} \Upsilon_{\text {eff }}
$$

and effective mass

$$
M=m_{0}\left(1+\frac{\Upsilon_{v}}{6} \tanh ^{\frac{3}{2}} \frac{T_{D}}{2 T}\right)=m_{0}\left(1+\frac{\Upsilon_{\mathrm{eff}}}{6} \tanh \frac{T_{D}}{2 T}\right)
$$

where the renormalized electron-phonon coupling constant is:

$$
\Upsilon_{\text {eff }} \equiv \Upsilon_{v} \tanh ^{\frac{1}{2}} \frac{T_{D}}{2 T}
$$

The polaron characteristics versus thermal energy are depicted in the Figures (Figure 1 self-action potential and Figure 2, the effective mass).

Figure 1 shows decrease self-energy for increase temperature and achieves the value zero for further temperature increase. This phenomenon is confirmed by Figure 2 where the effective mass decreases for increase temperature and for further increase temperature it achieves the value unity (value for the electron band mass). These results are in agreement with literature [16] [17] [18] [19]. The monotonic decrease of polaron characteristics with increase thermal energy confirms the renormalized electron-phonon coupling constant being tailored by the thermal energy where low temperatures favour auto-localization (quantum phase) of the carrier while high temperatures favour polaron undressing and subsequent quenching of the quantum behaviour rendering the system classical. The situation is well illustrated in Figure 2 where the effective mass achieves the quantum-phase values for low temperature and band electron mass for high temperature highlighting a phase transition. It is instructive to note that very small thermal energy is required remove the carrier in the quantum phase. From Figure 1 and Figure 2 we observe the critical temperature $T=\tau_{C}$ (phase transition temperature) above which $\left(T \gg \tau_{c}\right)$, the polaron characteristics have a monotonic decreases that the system loses its quantum phase and consequently a grip on the dynamical polarization in the phonon cloud as expected and achieves the classical phase with self-energy potential energy

$$
U_{\Upsilon}=0,
$$

and the effective mass achieving the electron band mass

$$
\lim _{T \rightarrow+\infty} M=m_{0}
$$




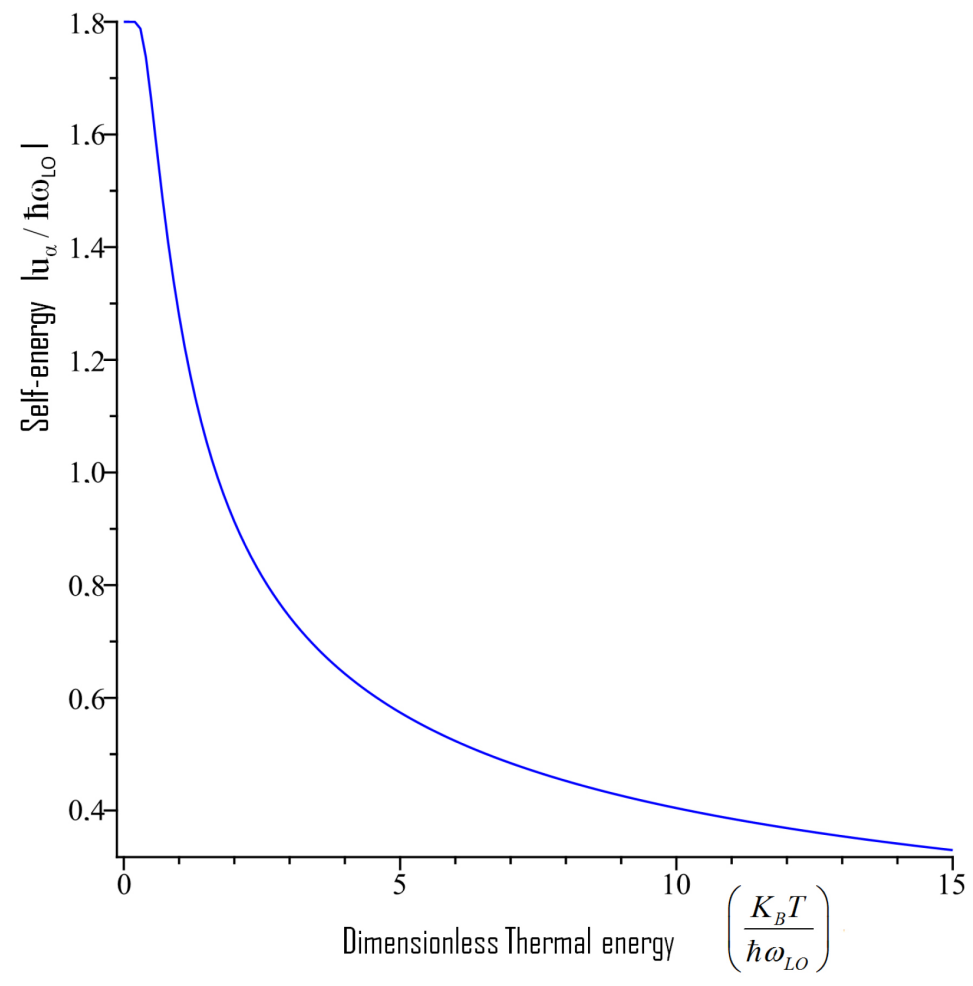

Figure 1. Plot of self-energy $\left|U_{\Upsilon} / \hbar \omega_{L O}\right|$ in units of the phonon energy versus thermal energy for weakly coupled system.

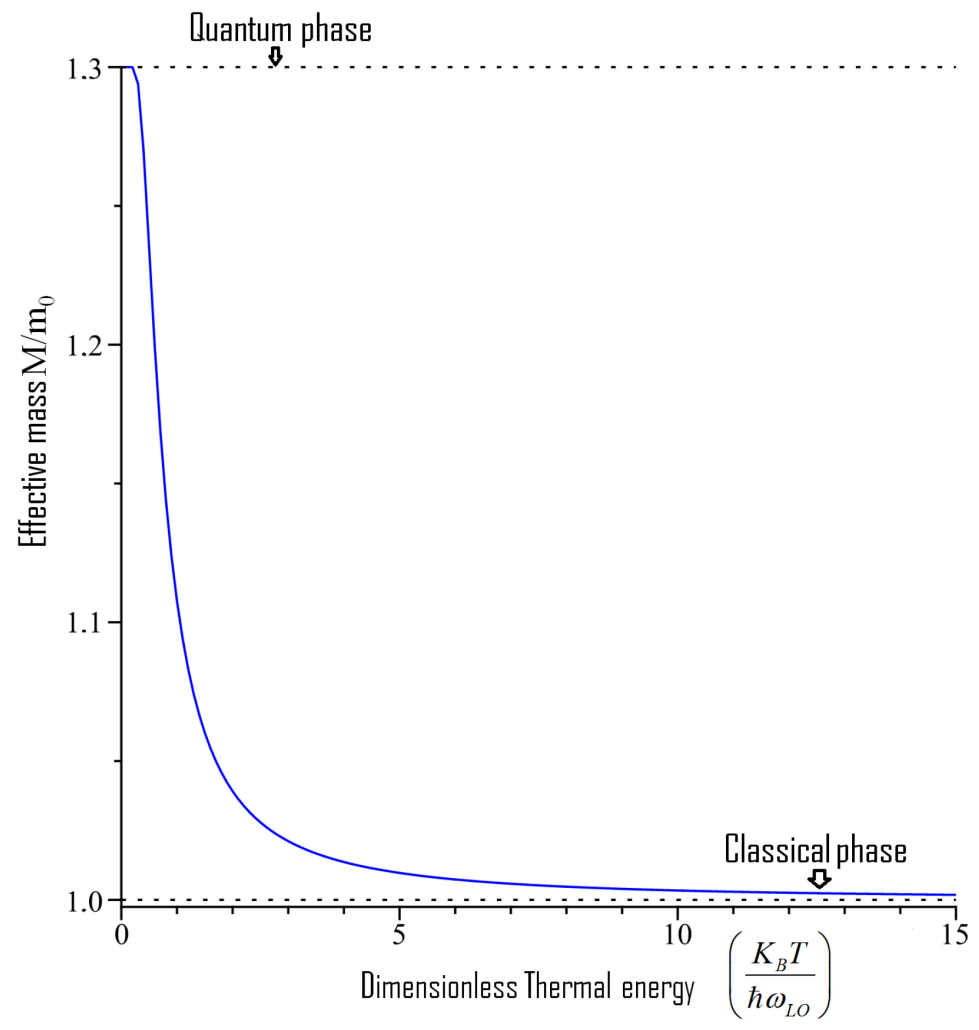

Figure 2. Plot of effective mass $\left|M / m_{0}\right|$ in units of the electron band mass versus thermal energy for weakly coupled system. 
and below which ( $T \ll \tau_{c}$ ), the coupling between the electron and the dynamical polarization in the phonon cloud grows non-perturbatively so that the self-action potential energy achieves the(quantum phase) value

$$
\lim _{T \rightarrow 0} \frac{u_{\Upsilon}}{\hbar \omega_{L O}}=-\Upsilon_{v}
$$

and the effective mass

$$
\lim _{T \rightarrow 0} \frac{M}{m_{0}}=1+\frac{\Upsilon_{v}}{6} .
$$

The latter correspond to the Polaron characteristics in the quasi-free state. Therefore, by enhancing the thermal energy of a weakly coupled system, the Polaron characteristics decay quickly and achieve the classical phase characterized by free-electron state or undress polarons.

\subsection{Strong-Coupling Limit}

Extremising the expression in (12) with respect to the variational parameter $\gamma_{Q}$ for low temperatures $T<\tau_{c}$, the polaron self-action potential energy achieves the form

$$
U_{\Upsilon}=-0.10610 \hbar \omega_{L O} \Upsilon_{v}^{2} \operatorname{erf}\left(\Upsilon_{v}^{2} \tanh \left(\frac{\beta \vartheta}{2}\right)\right)^{4}
$$

and the effective mass

$$
M=m_{0}\left(1+0.02001 \Upsilon_{v}^{4} \operatorname{erf}\left(\Upsilon_{v}^{2} \tanh \left(\frac{\beta \vartheta}{2}\right)\right)^{12}\right)
$$

Here, the probability integral with argument $Z$ is defined as:

$$
\operatorname{erf}(z)=\frac{2}{\sqrt{\pi}} \int_{0}^{z} \exp \left\{-t^{2}\right\} \mathrm{d} t .
$$

For exceedingly low temperatures $T \ll \tau_{c}$ then the polaron self-energy and effective mass achieves respectively the (quantum-phase) values

$$
\begin{aligned}
& U_{\Upsilon}=-0.10610 \hbar \omega_{L O} \Upsilon_{v}^{2} \\
& M=m_{0}\left(1+0.02001 \Upsilon_{v}^{4}\right)
\end{aligned}
$$

These are respectively the polaron energy and effective mass for the 3D strong-coupling regimes [6] [7] [27] [31] [32] and characterizing self-trapped states. For high temperatures, the polaron characteristics are obtained from relations (14) and (15) respectively as

$$
\begin{gathered}
U_{\Upsilon}=-\hbar \omega_{L O} \Upsilon_{\text {eff }} \\
M=m_{0}\left(1+\frac{\Upsilon_{\text {eff }}}{6}\right)
\end{gathered}
$$

And mimics the Polaron characteristics in quasi-free states (see section 3.1). For exceedingly high temperatures $T \gg \tau_{c}$

$$
U_{\Upsilon}=0, M=m_{0} .
$$

The quasi-particle achieves bare electron characteristics referring the system 
classical (undressed polaron). The latter mimics exactly the results for the weak coupling regime. The plot of the polaron energy versus temperature is depicted on Figure 3 and the effective mass on Figure 4.

Figure 3 depicts the variations of the self-action potential as a function of thermal energy while Figure 4 depicts variations of the Polaron effective mass with thermal energy. The self-action as well as the effective mass shows a monotonic decrease with increase temperature [16] [17] [18] [19] above the critical value:

$$
\tau_{c}=\frac{T_{D}}{2 \operatorname{arctanh}\left(\frac{\Upsilon_{c}^{2}}{\Upsilon_{v}^{2}}\right)}
$$

Here, the Debye temperature is and the critical coupling constant characterizes the value with enhanced self-trapping. This critical temperature should be the crossover temperature below which the coupling of the electron to the dynamical polarization in the phonon cloud grows non-perturbatively. From the plot of the polaron energy versus the coupling constant, for exceedingly high temperatures $T \gg \tau_{C}$, the energy has a linear behaviour (renormalized by the hyperbolic tangent function) as in the weak-coupling regime ( quasi-free states) while for low temperatures it has a parabolic behaviour as in the strong-coupling regime and imitate results observed in literature [7] [27] [31] [32] (self-tapped states). The effective mass has a four-power law for the low temperature regime while for the high temperature regime, a linear law renormalized by the hyperbolic tangent function of the thermal energy.

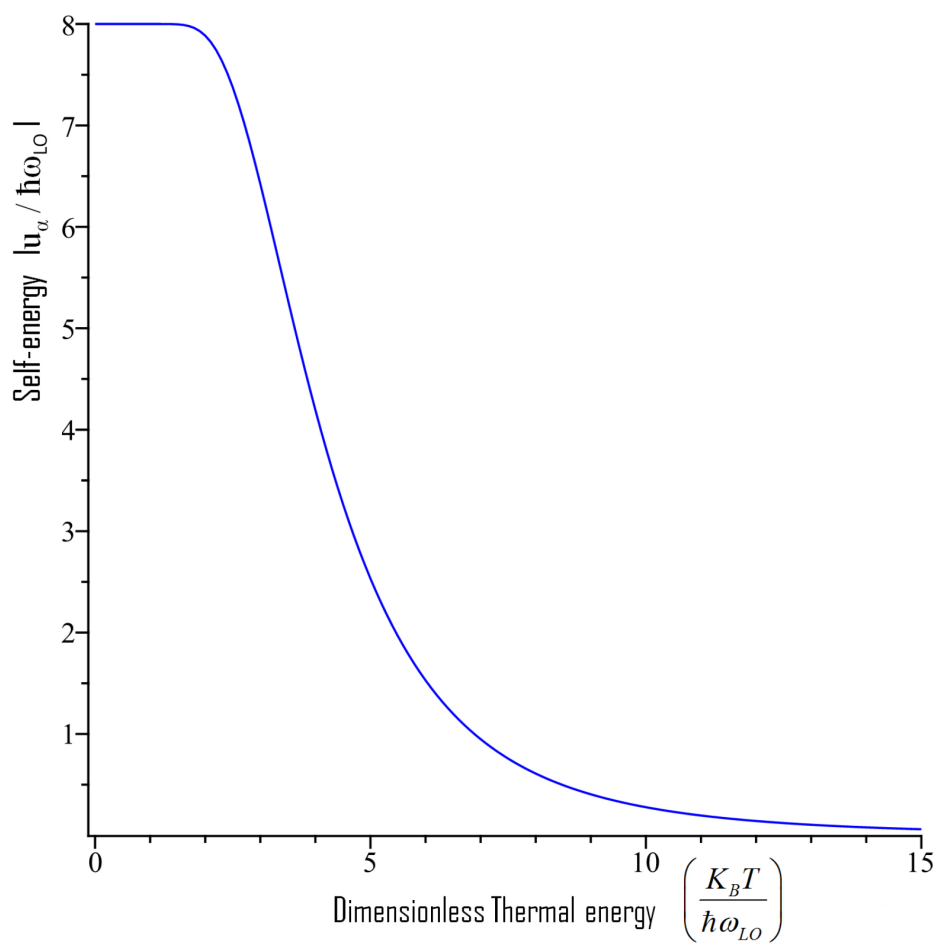

Figure 3. Plot of self-energy $\left|U_{\curlyvee} / \hbar \omega_{L O}\right|$ in units of the phonon energy versus thermal energy for strongly coupled system. 




Figure 4. Plot of effective mass $\left|M / m_{0}\right|$ in units of the electron band mass versus thermal energy for strongly coupled system.

The observation of Figure 3 and Figure 4 shows that in contrast with weakly coupled system, the quantum phase (self-trapping) is being kept longer. A high thermal energy is required to destroy the quantum phase and then produce phase transition. The variation of the thermal energy makes the quasi-particles to changes its characteristics. The quantum behaviour (self-trapping) survive for low temperature and quenches for high temperature (classical phase). That illustrate the self-trapping transition characterised by the quenching of auto-localisation in Polaron states. This general result has been confirmed experimentally for exciton in $M_{n}{ }^{2+}$ doped ethylammonium lead bromide where critical temperature above which band edge and self-trapped exciton emission are suppressed is estimated [20].

\section{Conclusions}

The paper, investigates the thermal energy effect on electron auto-localization and finds the critical temperature $\tau_{C}$ characterizing the limit below which the polaron is self-trapped. The polaron characteristics (self-action potential energy and effective mass) are observed to be expressed via the renormalized electron-phonon coupling constant tailored by the thermal energy. Low temperatures are observed to favour auto-localization of the carrier while high temperatures favour polaron undressing and subsequent quenching of the quantum behaviour thereby rendering the system classical. The critical (transition) temper- 
ature expressed via the critical coupling constant is observed to be the transition point between the quantum and the classical phases. This critical temperature should be the crossover temperature below which the coupling between the electron and the dynamical polarization in the phonon cloud grows non-perturbatively. So, for temperatutres greater than the critical temperature, the system has the tendency to lose the grip on the dynamical polarization in the phonon cloud and for exceedingly high temperatures, the energy has a linear behaviour (renormalized by the hyperbolic tangent function) as in the weak-coupling regime while for low temperatures, it has a parabolic behaviour as in the strong-coupling regime. The effective mass has a four-power law for the low temperature regime while for the high temperature, a linear law renormalized by the hyperbolic tangent function of the thermal energy.

Therefore, even though many authors predict that the Polaron do not undergoes phase transition, it is demonstrated that, in the particular case of external influence (thermal energy), the electro-phonon coupling is renormalized and the phase transition is observed (from self-trapped to quasi-free states).

\section{Acknowledgements}

This research did not receive any specific grants from funding agencies in the public, commercial, or not for profit sectors.

\section{Data Availability Statement}

The data that supports the findings of this study are available within this article.

\section{Conflicts of Interest}

The authors declare no conflicts of interest regarding the publication of this paper.

\section{References}

[1] Stoneham, A.M., Gavartin, J., Shluger, A.L., Kimmel, A.V., Ramo, D.M., Rønnow, H.M., Aeppli, G. and Renner, C. (2007) Trapping, Self-Trapping and the Polaron Family. Journal of Physics: Condensed Matter, 19, Article ID: 255208. https://doi.org/10.1088/0953-8984/19/25/255208

[2] Lany, S. and Zunger, A. (2009) Polaronic Hole Localization and Multiple Hole Binding of Acceptors in Oxide Wide-Gap Semiconductors. Physical Review B, 80, Article ID: 085202. https://doi.org/10.1103/PhysRevB.80.085202

[3] Paleari, A., Meinardi, F., Brovelli, S. and Lorenzi, R. (2018) Competition between Green Self-Trapped-Exciton and Red Non-Bridging-Oxygen Emissions in $\mathrm{SiO}_{2}$ under Interband Excitation. Communication Physics, 1, Article No. 67. https://doi.org/10.1038/s42005-018-0069-5

[4] Malyukina, Y.V. and Sorokin, A.V. (2016) Features of Exciton Dynamics in Molecular Nanoclusters (J-Aggregates): Exciton Self-Trapping. Low Temperature Physics, 42, 429-440. https://doi.org/10.1063/1.4955493

[5] Landau, L.D. (1933) Electron Motion in Crystal Lattices. Physikalische Zeitschrift der Sowjetunion, 3, 664. 
[6] Lang, I.G. and Firsov, Y.A. (1963) Kinetic Theory of Semiconductors with Low Mobility. Soviet Journal of Experimental and Theoretical Physics, 16, 1301.

[7] Pekar, S.I. (1946) Local Quantum States of Electrons in an Ideal Ion Crystal ZhurnalEksperimentalnoi I TeoreticheskoiFiziki, 10, Article No. 341.

[8] Pekar, S.I. (1946) The Method of Effective Masses of Electrons in Crystals. ZhurnaIEksperimentalnoi I TeoreticheskoiFiziki, 16, Article No. 933.

[9] Pekar, S.I. (1963) Electron Theory of Crystals with Ionic Lattices-Dielectrics and Semiconductors. ZhurnalEksperimentalnoi I TeoreticheskoiFiziki, 16, Article No. 341.

[10] Frohlich, H. (1954) Electrons in Lattice Fields. Advances in Physics, 3, 325-361. https://doi.org/10.1080/00018735400101213

[11] Fantoni, R. (2012) Localization of Acoustic Polarons at Low Temperatures: A Path-Integral Monte Carlo Approach. Physical Review B, 86, Article ID: 144304. https://doi.org/10.1103/PhysRevB.86.144304

[12] Hou, J. and Si, G. (2014) Acoustic Polaron in Free-Standing Slabs. Journal of Condensed Matter Physics, 4, 235-240. https://doi.org/10.4236/wjcmp.2014.44025

[13] Peeters, F.M. and Devreese, J.T. (1895) Acoustical Polaron in Three Dimensions: The Ground-State Energy and the Self-Trapping Transition. Physical Review B, 32, Article No. 3515. https://doi.org/10.1103/PhysRevB.32.3515

[14] Hou, J.H. and Liang, X.X. (2007) On the Possibility of Self-Trapping Transition of Acoustic Polarons in Two Dimensions. Chinese Physics, 16, Article No. 3059. https://doi.org/10.1088/1009-1963/16/10/040

[15] Whitfield, G. and Engineer, M. (1975) Temperature Dependence of the Polaron. Physical Review B, 12, Article No. 5472. https://doi.org/10.1103/PhysRevB.12.5472

[16] Li, Y.C. and Gu, S.W. (1989) Polarons in a Polar Crystal Slab at Finite Temperatures. Journal of Physics: Condensed Matter, 12, Article No. 3201. https://doi.org/10.1088/0953-8984/1/20/003

[17] Wu, X.G., Peeters, F.M. and Devreese, J.T. (1987) Temperature Dependence of the Polaron Mass in a GaAs-AlxGa1-xAs Heterostructure. Physical Review B, 36, 9765-9768. https://doi.org/10.1103/PhysRevB.36.9765

[18] Eerdunchaolu, Wuyunqimuge, and Han, C. (2011) Temperature Dependence of Effective Mass of Weak-Coupling Polaron in a Quantum Rod. Acta Optica Sinica, 31, 3788 .

[19] Eerdunchaolu and Xiao, J.L. (2007) Effects of Lattice Vibration on the Properties of the Strong-Coupling Polaron in a Quantum Well. Journal of the Physical Society of Japan, 76, Article ID: 044702. https://doi.org/10.1143/JPSJ.76.044702

[20] Sarang, S., Delmas, W., Naghadeh, S.B., Cherrette, V., Zhang, J.Z. and Ghos, S. (2020) Low-Temperature Energy Transfer via Self-Trapped Excitons in $\mathrm{Mn}^{2+}$-Doped 2D Organometal Halide Perovskites. Journal of Physical Chemistry Letters, 11, 10368-10374. https://doi.org/10.1021/acs.jpclett.0c03287

[21] Gerlach, B. and Löwen, H. (1991) Analytical Properties of Polaron Systems or: Do Polaronic Phase Transitions Exist or Not? Reviews of Modern Physics, 63, 63-90. https://doi.org/10.1103/RevModPhys.63.63

[22] Peeters, F.M. and Devreese, J.T. (1982) On the Existence of a Phase Transition for the Fröhlich Polaron. Physica Status Solidi (B), 112, 219-229.

https://doi.org/10.1002/pssb.2221120125

[23] Wei, B.-H., Zhao, X.-J. and Gu, S.-W. (1990) Magnetic Field and Temperature Dependence of the Self-Trapping Energy of a Polaron in a Polar-Crystal Slab in Arbi- 
trary Magnetic Field Strength. Physical Review B, 41, 1368-1375. https://doi.org/10.1103/PhysRevB.41.1368

[24] Yu, Y., Ma, L., Cai, P., Zhong, R., Ye, C., Shen, J., Gu, G.D., Chen, X.H. and Zhang, Y. (2019) High-Temperature Superconductivity in Monolayer $\mathrm{Bi}_{2} \mathrm{Sr}_{2} \mathrm{CaCu}_{2} \mathrm{O}_{8+\delta}$. Nature, 575, 156-163. https://doi.org/10.1038/s41586-019-1718-x

[25] Frost, J.M. (2017) Calculating Polaron Mobility in Halide Perovskites. Physical Review B, 19, Article ID: 195202. https://doi.org/10.1103/PhysRevB.96.195202

[26] Hou, J.H. and Liang, X.X. (2007) Self-Trapping of Acoustic Polaron in One Dimension. Chinese Physics Letters, 24, Article No. 3222. https://doi.org/10.1088/0256-307X/24/11/055

[27] Jipdi, M.N., Fokou, I.F., Tchoffo, M. and Fai, L.C. (2019) Optical and Acoustic Polaron Formation: Dynamic Matrix Approach (DMA). The European Physical Journal Plus, 134, Article No. 525. https://doi.org/10.1140/epip/i2019-12902-9

[28] Jipdi, M.N., Tchoffo, M. and Fai, L.C. (2018) Adiabatically Modeling Quantum Gates with Two-Site Heisenberg Spins Chain: Noise vs Interferometry. Physica E: Low-Dimensional Systems and Nanostructures, 96, 36-45.

https://doi.org/10.1016/j.physe.2017.09.023

[29] Jipdi, M.N., Fai, L.C. and Tchoffo, M. (2016) Quantum System Driven by Incoherent a.c Fields: Multi-Crossing Landau Zener Dynamics. Physics Letters A, 380, 3665-3671. https://doi.org/10.1016/j.physleta.2016.08.044

[30] Fai, L.C., Tchoffo, M. and Jipdi, M.N. (2015) Multi-Particle and Multi-State Landau-Zener Model: Dynamic Matrix Approach. European Physical Journal Plus, 130, Article No. 71. https://doi.org/10.1140/epjp/i2015-15071-y

[31] Landau, L.D. and Pekar, S.I. (1948) Effective Mass of a Polaron. ZhurnalEksperimentalnoi I TeoreticheskoiFiziki, 18, 419-423.

[32] Devreese, J.T. (2003) Polarons. AIP Conference Proceedings, 678, Article No. 3. https://doi.org/10.1063/1.1612391 